\title{
A Young Stellar Cluster in the Nucleus of NGC 4449
}

\author{
Torsten Böker ${ }^{1}$, Roeland P. van der Marel \\ Space Telescope Science Institute, 3700 San Martin Drive, Baltimore, MD 21218 \\ boeker@stsci.edu, marel@stsci.edu \\ Lisa Mazzuca ${ }^{2}$ \\ Goddard Space Flight Center, Greenbelt, MD 20771 \\ lmazzuca@pop500.gsfc.nasa.gov \\ Hans-Walter Rix, Gregory Rudnick \\ Max-Planck-Institut für Astronomie, Königstuhl 17, D-69117 Heidelberg, Germany \\ rix@mpia-hd.mpg.de, rudnick@mpia-hd.mpg.de \\ Luis C. Ho \\ Observatories of the Carnegie Institution of Washington, 813 Santa Barbara Street, Pasadena, \\ CA 91101-1292 \\ lho@ociw.edu \\ Joseph C. Shields \\ Ohio University, Department of Physics and Astronomy, Clippinger Research Laboratories, 251B, \\ Athens, $\mathrm{OH} 45701-2979$ \\ shields@helios.phy.ohiou.edu
}

\begin{abstract}
We have obtained 1-2 $\AA$ resolution optical Echellette spectra of the nuclear star cluster in the nearby starburst galaxy NGC 4449. The light is clearly dominated by a very young $(6-10 \mathrm{Myr})$ population of stars. For our age dating, we have used recent population synthesis models to interpret the observed equivalent width of stellar absorption features such as the H I Balmer series and the Ca II triplet around $8500 \AA$. We also compare the observed spectrum of the nuclear cluster to synthesized spectra
\end{abstract}

\footnotetext{
${ }^{1}$ Affiliated with the Astrophysics Division, Space Science Department, European Space Agency.

${ }^{2}$ Current address: Department of Astronomy, University of Maryland, College Park, MD 20742
} 
for stellar populations of varying ages. All these approaches yield a consistent cluster age. Metallicity estimates based on the relative intensities of various ionization lines yield no evidence for significant enrichment in the center of this low mass galaxy: the metallicity of the nuclear cluster is about one fourth of the solar value, in agreement with independent estimates for the disk material of NGC 4449.

Subject headings: galaxies: individual (NGC 4449) — galaxies: starburst — galaxies: nuclei.

\section{Introduction}

Recent observations with the Hubble Space Telescope (HST) at both optical (Phillips et al. 1996; Matthews et al. 1999) and near-infrared (NIR, Carollo, Stiavelli, \& Mack 1998; Böker et al. 1999a) wavelengths have revealed that a compact, photometrically distinct star cluster is often present in the center of spiral galaxies of all Hubble types. The formation mechanism of such clusters is puzzling, especially in late-type spirals which by definition do not possess a prominent bulge. In these cases, the gravitational potential is very shallow, and it is far from obvious how the gas could fall all the way to the center to form a dense star cluster.

In this paper, we describe the results of a pilot program to investigate the processes of nuclear cluster formation. More specifically, we have performed 1-2 $\AA$ resolution optical Echellette spectroscopy of the nucleus of NGC 4449, which is part of a small sample of galaxies with prominent nuclear star clusters that were identified from a recent NIR survey with HST (Böker et al. 1999a). The morphological classification of NGC 4449 has been the subject of some confusion. It was originally classified as Irr by Hubble (1936) and Sandage (1961), but it is listed as Sm in Sandage \& Tamman (1981). As van den Bergh (1995) points out, the presence of a stellar nucleus might be a useful criterion to distinguish between the two classes, since Irregulars usually do not have a well-defined nucleus - in contrast to late-type spirals, as discussed above. The extent, morphology, and dynamics of its H I gas suggest that NGC 4449 has undergone some interaction in the past, a fact that might well account for its unusually high star formation activity (Hunter, van Woerden \& Gallagher 1999). It also has a prominent stellar bar which covers a large fraction of the optically visible galaxy. Both the nuclear cluster and the stellar bar are evident in Figure 1, which shows a ground-based R-band image by Frei et al. (1996).

The fact that the nucleus of NGC 4449 is also prominently seen in the far ultraviolet (FUV) (Hill et al. 1998) and in various emission lines (Sabbadin et al. 1984; Mayya 1994; Hill et al. 1998; Böker et al. 1999a) indicates that it is the site of ongoing star formation. NGC 4449 is somewhat unusual in this regard, since emission-line surveys have shown that the nuclei of late-type galaxies often are devoid of ionized gas (e.g. Böker et al. 1999a). It appears that NGC 4449 is one of the few late-type spirals that is "caught in the act" of ongoing star formation in its nucleus.

NGC 4449 was chosen for a case study because of its high cluster-to-disk contrast which allows

us to isolate the light of the nuclear cluster from the underlying galaxy. From the disk-subtracted 
cluster spectrum, we derive the equivalent width (EW) of a number of stellar absorption features. We then use stellar population synthesis methods to constrain the star formation history of the nuclear cluster and to determine the age of its dominant stellar population. In $\S 2$, we describe our observations and the data reduction methods. Section 3 describes the quantitative analysis of a number of emission lines and stellar absorption features, as well as our spectral synthesis efforts. From the results of these various diagnostic tools, we deduce an age of about $6-10$ Myr for the nuclear cluster. In $\S 4$, we discuss our results and compare them to a recent study by Gelatt, Hunter, \& Gallagher (2001) which confirms our age estimate from a complementary dataset. We also briefly discuss suggested mechanisms of nuclear cluster formation and their possible impact on the evolution of the host galaxy.

\section{Observations and data reduction}

The data described here were taken in April 1999 at the Steward Observatory 90" Bok reflector. We used the Boller \& Chivens spectrograph in Echellette mode with a $2^{\prime \prime}$-wide slit to obtain spectra with $1-2 \AA$ resolution covering the range $\approx 3600-9000 \AA$. The average seeing over the observation was $2.5^{\prime \prime}$.

Following Willmarth \& Barnes (1994), we used standard IRAF ${ }^{3}$ routines in the LONGSLIT and ECHELLE packages to perform the following data reduction steps. After the data frames were bias-subtracted and trimmed using the $C C D P R O C$ routine, we corrected for bad pixels. We obtained a bad pixel mask by ratioing two domeflat exposures with different integration times, and replaced the bad pixels with a median filter. A second pass through CCDPROC then flatfielded the data using a median-combined set of domeflat exposures.

We obtained three 30 minute exposures of NGC 4449 which were median-combined after verifying that the brightness profile was properly centered on the slit for all three exposures. Using the APALL task, we then extracted the multi-order spectrum of the galaxy over 12 subapertures of $1^{\prime \prime}$ each, thus retaining the spatial information. Spectra of the standard star Hiltner 600 were extracted with the DOECSLIT task and used to measure the Echelette sensitivity function, based on spectrophotometry published by Massey et al. (1988). Also within DOECSLIT, we determined the dispersion correction from spectra of a Helium-Argon lamp. Both flux and wavelength calibration were then applied to the galaxy spectrum, before we used SCOMBINE to combine all orders into one two-dimensional spectrum covering the full wavelength range.

Finally, we averaged the central $2^{\prime \prime}$ into a one-dimensional spectrum of the nucleus, as well as two stripes of $3^{\prime \prime}$ width each, located $3.5^{\prime \prime}$ on either side of the nucleus. Subtracting the two resulting 1-d spectra from each other eliminates the sky emission lines and isolates the light of the nuclear star cluster from the underlying galaxy disk. The simple linear interpolation of the underlying disk is likely to leave a small residual contribution to the spectrum of the nuclear cluster.

\footnotetext{
${ }^{3}$ IRAF is distributed by the National Optical Astronomical Observatories, which are operated by AURA, Inc. under contract to the NSF.
} 
Higher order interpolation clearly is desirable, but proved unfeasible over the full wavelength range of the spectrum. However, for limited wavelength ranges, we verified that the results of our analysis are robust against such variations in the methodology of the background subtraction. We conclude that the errors introduced by the imperfect interpolation of the galaxy surface brightness profile are small.

As the result of the described data reduction process, the flux- and wavelength-calibrated spectrum between 3600 and $9000 \AA$ of the central $2^{\prime \prime}$ of NGC 4449 is shown in Figure 2. The red end of the spectrum is subject to some residual fringing with peak-to-peak amplitudes smaller than $10 \%$ of the continuum. This slightly affects the uncertainty of the the continuum level for measurement of the Ca triplet absorption lines (see $\S 3.1 .2$ ). The large residuals near $7580 \AA$ are due to imperfect correction of telluric oxygen absorption ("A-band"), as are smaller residuals at $6280 \AA$ and $6860 \AA$.

\section{Results}

\subsection{Stellar Absorption Features}

In order to measure the equivalent width (EW) of various stellar absorption features, we rectified and normalized the spectrum. The line and continuum windows that we used for continuum fitting and EW measurement are defined in González Delgado, Leitherer \& Heckman (1999) for the Balmer and HeI series, and in Díaz, Terlevich \& Terlevich (1989) for the CaII triplet (CaT) around $8500 \AA$. They are listed in Table 1, together with all measured EWs. Figure 3 shows the rectified spectrum over two exemplary regions which contain some higher order Balmer lines and the CaT, respectively. The analysis of the Balmer absorption is complicated by the presence of emission lines. We took the following approach to subtract the line emission before measuring the EW of the absorption features. We iteratively subtracted Gaussian components (typically one or two) until the resulting "pure" absorption spectrum of each Balmer line could be well fitted by a Voigt-profile. Figure 4 demonstrates the process. The sum of the fitted Gauss components then yields the emission line fluxes for the Balmer series. Fitting synthesized spectra of stellar populations as described in $\S 3.2$ yields an alternative approach. After subtraction of the best fitting model spectrum, the residuals contain only the line emission. The line fluxes derived with both methods are consistent. We conservatively estimate the uncertainty in the derived EWs of the Balmer absorption to be $10 \%$. In what follows, we compare the measured EWs to evolutionary synthesis models.

\subsubsection{Balmer absorption}

In Figure 5, we compare our measurements to evolutionary synthesis models of González Delgado et al. (1999). Each panel contains the time evolution of the EW of a specific absorption 
feature. The grey horizontal bar denotes the respective measured EW, the width of the bar indicates the uncertainty of $10 \%$. The crossing point of the bar with the model yields a solution for the cluster age. All features yield an age of $6-10 \mathrm{Myr}$ for the dominant stellar population in the nuclear cluster of NGC 4449. This result is rather independent of the detailed IMF or the star formation mode (continuous or instantaneous). For all reasonable models, the rise in the Balmer EW occurs at about the same time, so that the crossing point of the measurements with the model curve is robust to within a few million years. A theoretical second solution, namely an extremely old cluster with an age around 10 Gyr is ruled out from the evidence that follows.

\subsubsection{Calcium triplet}

Because the CaT absorption is produced in cooler and more evolved stars than the Balmer series, it provides an independent age estimator. In Figure 3b, we show the spectral region comprising the CaII triplet around $8500 \AA$ which also contains the line and continuum windows used for our analysis. These are also listed in Table 1 and are identical to those used by García-Vargas, Mollá \& Bressan (1998) to derive theoretical EWs of the CaT from evolutionary synthesis models.

We compare the EW sum of the two strongest features ( $\lambda 8542$ and $\lambda 8662)$ as measured from our data to the García-Vargas et al. (1998) models (their grid I) in Figure 6. The horizontal line again denotes the measured EW of the CaT and the shaded region its estimated uncertainty which is dominated by the uncertainty in the continuum level due to residual fringing on the red end of the spectrum. Despite the conservative error estimate, the very deep CaT absorption in the nuclear cluster of NGC 4449 unambiguously confirms a young cluster age between 5 and 20 Myr.

Figure 6 also seems to indicate a high metallicity of at least solar value. However, we caution that the low-metallicity stellar evolutionary tracks used in the population synthesis models have been shown to systematically underpredict the red supergiant (RSG) features (Origlia et al. 1999). This is a longstanding problem that also manifests itself in the inability of current models to reproduce the observed blue-to-red supergiant ratio in galaxies (e.g. Langer \& Maeder 1995). As a consequence, the predicted CaT EW for low-metallicity RSG populations falls significantly below the observed values in, e.g., young clusters in the LMC (Bica, Santos, \& Alloin 1990). For individual stars, the CaT EW exceeds 9 Åonly in red supergiants (Díaz et al. 1989). Certainly, any single-age population of stars that has a CaT EW as high as the nuclear cluster of NGC 4449 has to contain red supergiants with ages between 5 and $20 \mathrm{Myr}$, irrespective of its metallicity. In summary, Figure 6 confirms a young cluster age, but does not provide a reliable way to constrain its metallicity. In $\S 3.3 .2$, we will explore alternative methods to derive the metallicity based on nebular emission lines.

\section{2. $\quad$ Spectral modeling}

As an additional test of the above results we have compared the spectrum of the nuclear cluster in NGC 4449 to spectral models, using the entire available wavelength range. For this we 
first created model spectra of simple stellar populations (SSPs), i.e. populations that form in instantaneous, short-lived starbursts. Models were calculated for ages on a logarithmically spaced grid with a spacing of 0.5 dex. We used the 1996 version of the Bruzual \& Charlot (1993) models, which assume a Salpeter (1955) IMF between 0.1 and $125 \mathrm{M}_{\odot}$ and solar metallicity. The observed spectrum is then modeled as a linear superposition of these SSPs, which essentially allows an arbitrary star formation history (approximated as a weighted sum of delta functions). We find the weights that optimize the fit to the data, allowing also for a redshift due to the systemic velocity of the galaxy. The $\chi^{2}$ fit was performed with the software of Rix \& White (1992), developed originally for the analysis of galaxy kinematics.

The best fit to the observed spectrum was obtained with a model that has the large majority of its light $(>90 \%)$ in the SSP of age $10^{7}$ years. The data-model comparison is shown in Figure 7. The overall shape of the spectrum as well as the depth of the Balmer absorption features are well reproduced. However, the Ca II feature at $3934 \AA$, as well as some other metal lines, are significantly underestimated in the fit. This discrepancy is possibly due to a metallicity mismatch. We used template spectra with solar abundances for the modeling because we did not have lower metallicity tracks with sufficient spectral resolution available. However, as we will show in $\S 3.3 .2$, the nuclear cluster in NGC 4449 does actually have subsolar metallicity. Nonetheless, the shape of the $4000 \AA$ break and the depth of the Balmer series absorption features depends only slightly on metallicity, so that the quality of the fit should yield a good age estimate despite the non-perfect choice of metallicity. We conclude that the spectral modeling confirms the results of our previous analysis that the nuclear cluster in NGC 4449 is young.

\subsection{Emission lines}

As can be seen from Figure 2, the spectrum of the nuclear star cluster of NGC 4449 - after subtraction of the circum-nuclear light - contains a number of strong emission lines. After subtracting the continuum fit described in $\S 3.2$, we measured the fluxes of all significant emission lines (except H9 and H10) using SPECFIT as implemented in IRAF (Kriss 1994). The residual spectrum was subdivided into three segments and a fit with $\chi^{2}$ optimization was performed, assuming a linear fit to any residual continuum and Gaussian profiles in a common velocity frame for the emission lines. The fluxes of $\mathrm{H} 9$ and $\mathrm{H} 10$ were measured as described in $\S 3.1$ to more accurately correct for the underlying absorption. The resulting fluxes on an absolute scale and relative to $\mathrm{H} \beta$ are listed in Table 2, along with their 1- $\sigma$ uncertainties.

\subsubsection{Extinction}

Extinction of the nebular emission can be quantified from reddening of the spectrum as indicated by the Balmer emission line ratios. For the intrinsic relative line strengths, we employed

predictions from Hummer \& Storey (1987) assuming Case B recombination in a $10^{4} \mathrm{~K}$ gas with 
$n_{e}=100 \mathrm{~cm}^{-3}$ (e.g., $\mathrm{H} \alpha / \mathrm{H} \beta=2.86$ ). The electron density can be constrained from the ratios of [O II] $\lambda 3729 / \lambda 3726=1.38 \pm 0.02$ and $[\mathrm{S} \mathrm{II}] \lambda 6716 / \lambda 6731=1.29 \pm 0.05$, both of which suggest $n_{e} \lesssim 100 \mathrm{~cm}^{-3}$ (e.g. Osterbrock 1989). For the extinction analysis, we employed the reddening law from Cardelli, Clayton \& Mathis (1989) with $R_{V}=3.1$. The measured Balmer emission fluxes ratioed to $\mathrm{H} \beta$ then imply extinction values of $\mathrm{A}_{\mathrm{V}}=(1.1,0.85,1.4,1.8,0.47,2.6)$ mag for $(\mathrm{H} \alpha, \mathrm{H} \gamma$, $\mathrm{H} \delta, \mathrm{H} \epsilon, \mathrm{H} 8, \mathrm{H} 9)$. The measured flux for $\mathrm{H} 8$ is almost certainly contaminated by emission in He I $\lambda 3890$, which would account for the low value of $A_{V}$ suggested by this line. The other features show a general trend of increasing $A_{V}$ with higher-order Balmer lines, which is probably an indication that the line fluxes are still slightly underestimated due to stellar absorption. We ultimately adopted an extinction value of $A_{V}=1.1 \mathrm{mag}$ based on the $\mathrm{H} \alpha / \mathrm{H} \beta$ ratio, since among the Balmer lines these features have the highest signal-to-noise ratio and are expected to suffer the least from underlying absorption. The majority of this extinction is evidently intrinsic to NGC 4449, since the foreground Galactic extinction is only 0.06 mag (Schlegel, Finkbeiner, \& Davis 1998). The $A_{V}$ adopted here is about one magnitude higher than the value derived by Ho, Filippenko \& Sargent (1997) using the same intrinsic line ratio. On the other hand, Ho et al. (1997) removed the stellar continuum by subtracting generic template spectra in order to measure the emission-line strengths. The difference of about $30 \%$ in the measured line ratio needed to reconcile the two measurements can probably be explained by any one or a combination of the following reasons: a slight mismatch of the continuum template in the Ho et al. (1997) analysis, the fact that they used a somewhat larger aperture, or differences in spectral resolution (which affect the ability to separate the emission core from the absorption trough).

\subsubsection{Metallicity}

The relative line fluxes can be used to obtain an independant estimate for the metallicity in the nucleus of NGC 4449. The "bright-line" method originally proposed by Pagel et al. (1979) makes use of the quantity

$$
\mathrm{R}_{23} \equiv\left(\mathrm{I}_{3727}+\mathrm{I}_{4959}+\mathrm{I}_{5007}\right) / \mathrm{H}_{\beta}
$$

as a measure of metallicity. For the NGC 4449 nucleus, the reddening-corrected line fluxes imply $\mathrm{R}_{23}=8.2 \pm 0.2$. A recent analysis illustrating the available calibrations of $R_{23}$ is provided by Kobulnicky, Kennicutt, \& Pizagno (1999). Their Figure 8 indicates that our measured value of $\mathrm{R}_{23}$ falls at or near the turnaround point between the low- and high-metallicity branches for this diagnostic; this result implies $12+\log (\mathrm{O} / \mathrm{H}) \approx 8.3-8.4$, or about one fourth of the solar value $(12+\log (\mathrm{O} / \mathrm{H})=8.9)$. This abundance for the nucleus is in good agreement with the average value for the disk of NGC 4449 of 8.32 reported by Lisenfeld \& Ferrara (1998). Abundances can be inferred directly if diagnostics of the nebular electron temperature are available. Table 2 lists a detection of the auroral [O III] $\lambda 4363$ line, which in combination with [O III] $\lambda \lambda 4959,5007$ can be used for this purpose. We used the dereddened measurements to calculate the $\mathrm{O} / \mathrm{H}$ abundance using the methods described in Oey \& Shields (2000). The large error bar on the [O III] $\lambda 4363$ flux translates into substantial uncertainties in the derived quantities, with a resulting temperature of $16700 \pm 3500 \mathrm{~K}$ and $12+\log (\mathrm{O} / \mathrm{H})=7.8 \pm 0.3$. The latter abundance is consistent at the $2 \sigma$ level with the result based on the $\mathrm{R}_{23}$ diagnostic. 


\subsection{Mass and color of the nuclear cluster}

Assuming a cluster age of $10 \mathrm{Myr}$, a simple estimate of the cluster mass can be obtained from its V-band luminosity. Our flux-calibrated spectrum after subtraction of the underlying galaxy light (Figure 2) yields $\mathrm{F}_{550 \mathrm{~nm}} \approx 3.5 \times 10^{-15} \operatorname{ergs~s}^{-1} \mathrm{~cm}^{-2} \AA^{-1}$, or $m_{V}=15.0$. This value can be compared to evolutionary synthesis models for single-age populations such as those in the Starburst99 package (Leitherer et al. 1999). For a Salpeter IMF with $m_{l o w}=1 \mathrm{M}_{\odot}$ and $m_{u p}=100 \mathrm{M}_{\odot}$, the observed cluster luminosity requires a mass of about $4 \times 10^{5} \mathrm{M}_{\odot}$ (after correction for $\mathrm{A}_{\mathrm{V}}=1.1$, for a distance ${ }^{4}$ to $\mathrm{NGC} 4449$ of $3.9 \mathrm{Mpc}$ and an age of $10 \mathrm{Myr}$ ). This cluster mass should represent a lower limit for two reasons. First, the distance to NGC 4449 is rather uncertain and has been estimated as high as $5.4 \mathrm{Mpc}$ (Kraan-Korteweg \& Tamman 1979). In addition, the IMF likely has a lower mass cutoff below the assumed $1 \mathrm{M}_{\odot}$. Adding low-mass stars does not change the cluster luminosity much, but significantly increases the total mass.

As an additional check of our results, we have measured the color of the nuclear cluster. The $(\mathrm{V}-\mathrm{I})$ color of the spectrum in Figure 2 is $(\mathrm{V}-\mathrm{I})=1.1$. Correcting for an extinction of $\mathrm{A}_{\mathrm{V}}=1.1$ as derived from the nebular emission lines ( $\S 3.3 .1$ ), the intrinsic color of the stellar population is $(\mathrm{V}-\mathrm{I})=0.6$. This value is most likely a lower limit, since at UV- and optical wavelengths, the stellar continuum is usually less reddened than the nebular emission lines (Calzetti 1997). Comparison with the Leitherer et al. (1999) models shows that a color of (V - I) $\geq 0.6$ is entirely consistent with a $6-10$ Myr old population.

The mass in the nuclear cluster implied by the stellar continuum can be compared with that inferred from the nebular flux. The extinction-corrected $\mathrm{H} \beta$ flux of $8.9 \times 10^{-14} \mathrm{ergs} \mathrm{s}^{-1} \mathrm{~cm}^{-2}$ corresponds to a total $\mathrm{H} \beta$ luminosity of $1.6 \times 10^{38} \mathrm{ergs} \mathrm{s}^{-1}$. For Case B recombination ( $\S 3.3 .1$ ), this luminosity requires that hydrogen-ionizing photons be produced and absorbed at a rate of $Q(\mathrm{H})=3.4 \times 10^{50} \mathrm{~s}^{-1}$ within the H II region. Comparison to the Starburst99 models shows that this is entirely consistent with a $10 \mathrm{Myr}$ old cluster with a mass of $4 \times 10^{5} \mathrm{M}_{\odot}$ and a metallicity of about one fourth of the solar value.

The dereddened He I $\lambda 5876 / \mathrm{H} \beta$ ratio of $0.10 \pm 0.01$ can be compared with the Case B prediction (Benjamin et al. 1999) of $0.14\left(\frac{\mathrm{He}^{+} / \mathrm{H}^{+}}{0.1}\right)$, where $\mathrm{He}^{+} / \mathrm{H}^{+}$is the ionic abundance ratio. The fact that the observed line ratio is close to the prediction implies that most of the helium within the nuclear H II region is singly ionized. This condition sets a strong limit on the age of the cluster, since relatively hot stars are required to maintain the helium ionization. The production rate of heliumionizing photons implied by the extinction-corrected He I $\lambda 5876$ flux is $2.6 \times 10^{49} \mathrm{~s}^{-1}$. For the above cluster parameters, this luminosity is consistent with the Starburst99 prediction at an age of $\sim 7 \mathrm{Myr}$.

\footnotetext{
${ }^{4}$ derived by assuming that NGC 4449 follows the Hubble flow with $\mathrm{H}_{0}=65 \mathrm{~km} \mathrm{~s}^{-1} \mathrm{Mpc}^{-1}$
} 


\section{Discussion and outlook}

The results of this study imply that NGC 4449 can be added to a growing list of late-type spiral galaxies that host a compact, young star cluster in their nucleus. While accurate spectroscopy and hence a reliable age estimate - of nuclear clusters is available for only a few objects such as the Milky Way (Krabbe et al. 1995), NGC 7793 (Shields \& Filippenko 1992), and IC 342 (Böker, van der Marel \& Vacca 1999b), photometric measurements show that many clusters are fairly blue, e.g. those in M 31, M 33, NGC 4242, or ESO 359-029 (Lauer et al. 1998; Matthews et al. 1999). This indicates the existence of a young $(\leq 100 \mathrm{Myr})$ stellar population in many of these nuclei.

From an HST survey of intermediate-type spirals, Carollo et al. (1998) find that virtually all galaxies with exponential bulges (i.e. bulges with an exponential surface brightness profile, as opposed to "power-law" or " $R^{1 / 4}$ " bulges) host compact nuclear clusters and that their luminosity correlates with that of the host galaxy. If one assumes that the mass-to-light ratio of the host galaxies as a whole is roughly the same for all objects, the most straightforward interpretation of this result is that more massive galaxies have more massive nuclear clusters. On the other hand, stellar populations fade with time (e.g. Bruzual \& Charlot 1993). Another possible interpretation therefore is that more massive galaxies host younger clusters. This intrinsic degeneracy makes age dating of nuclear clusters from photometric data alone impossible. Spectroscopic analysis is needed to investigate the age distribution of nuclear clusters and to decide whether their formation is a one time event or not. If nuclear clusters indeed form recurrently, the formation processes likely impact the morphological evolution of the host galaxy.

Of particular interest in this context is a model suggested by Friedli \& Benz (1993) and developed further by Norman, Sellwood \& Hasan (1996). These authors point out that build-up of a central mass concentration - e.g. a nuclear star cluster - can dissolve stellar bars, and lead to the formation of a bulge via collective bending instabilities (Raha et al. 1991; Merritt \& Sellwood 1994). The Norman et al. (1996) simulations show that only $5 \%$ of the combined disk and bar mass in a central concentration is sufficient to destroy a stellar bar on short timescales, leading to a bulge-like distribution of stars. In this scenario, repeated cycles of bar formation - gas infall - cluster formation - bar disruption will build the galaxy bulge over time. If true, the Hubble classification scheme could be naturally explained as an evolutionary sequence from late to early Hubble types.

In the case of NGC 4449, the cluster mass of (at least) $4 \times 10^{5} \mathrm{M}_{\odot}$ derived in $\S 3.4$ would be sufficient to destroy a bar with a mass up to $10^{7} \mathrm{M}_{\odot}$. The unusually prominent stellar bar in NGC 4449, which covers a large part of the optically visible galaxy, is probably much more massive than this value. This can be seen from the total luminosity of NGC 4449, which is listed in the RC3 (de Vaucouleurs et al. 1991) as $\mathrm{V}_{\mathrm{T}}^{0}=9.53$, which corresponds to $\mathrm{L}_{\mathrm{V}} \approx 2 \times 10^{9} \mathrm{~L}_{\odot, \mathrm{V}}$. Since the optical image is dominated by the stellar bar, and typical mass-to-light ratios are of order unity,

the bar mass should be $\approx 10^{9} \mathrm{M}_{\odot}$. The fact that it co-exists with the nuclear cluster is therefore not inconsistent with the Norman et al. (1996) scenario.

However, as pointed out before, NGC 4449 is a special case because of its unusually high overall star formation rate and its complex history with evidence for past interactions. The complex gas 
flows produced by a past merger might well explain why - different from most other late-type spirals (e.g. Böker et al. 1999a) - the nucleus of NGC 4449 contains large amount of ionized gas. Furthermore, there is no indication of an underlying older population of stars in the nuclear cluster of NGC 4449, as is the case, e.g., in the Milky Way. We point out, however, that for external galaxies where individual stars cannot be resolved, it is difficult to detect an old stellar population underlying a young starburst because the latter is dominating the luminosity (assuming similar masses for both bursts). It is somewhat unclear at this point whether the processes that govern the star formation in the nucleus of NGC 4449 are similar to those in "typical" late-type spirals, or whether NGC 4449 is an entirely different "special" case.

After the present paper had been finished, another study of the nuclear cluster in NGC 4449 appeared in the literature (Gelatt et al. 2001). Using ground-based spectroscopy of the ${ }^{12} \mathrm{CO}(2,0)$ and ${ }^{12} \mathrm{CO}(3,1)$ absorption features around $2.3 \mu \mathrm{m}$ and UVI colors from HST imaging, these authors derived a cluster age between 8 and $15 \mathrm{Myr}$, in good agreement with our estimate of $6-10 \mathrm{Myr}$. Assuming a somewhat lower extinction to the NGC 4449 nucleus than derived by us from the Balmer decrement ( $\S 3.3 .1)$, Gelatt et al. conclude that the color of the nuclear cluster is better matched by evolutionary tracks with a metallicity of $\mathrm{Z}=0.008$, somewhat higher than the average over the NGC 4449 disk. However, increasing the extinction by one magnitude (roughly the difference between our extinction and theirs) would easily move the location of the nuclear cluster in their Figure 3 within the range of the lower metallicity tracks. After accounting for the different extinction estimates, the derived V-I color of the nuclear cluster differs by about 0.2 magnitudes in the two studies. This could be the result of the somewhat different aperture sizes used to extract the cluster light (1.4" for Gelatt et al. compared to $2.0^{\prime \prime}$ for our study). Either way, both papers come to very similar conclusions on the age of the nuclear cluster in NGC 4449, with complementary methods.

This study is part of a larger observational program that contains three independent, but related approaches. First, we will use the WFPC2 onboard the Hubble Space Telescope during Cycle 10 to measure the structural properties of all nuclear clusters found in a large sample of nearby, face-on spiral galaxies of late Hubble type. We will then measure the stellar velocity dispersion in the brightest nuclei found in order to derive the cluster mass, as recently demonstrated for the example of IC 342 (Böker et al. 1999b). Finally, we hope to obtain optical spectra with HST/STIS to derive the stellar population and to constrain the formation history of individual nuclear clusters in much the same way as described in this paper. We aim to study a large number of nuclear clusters to allow a statistical analysis of their properties and dependence on the Hubble type of the host galaxy. Since ground-based observations are possible only for the brightest nuclear clusters, they are likely biased towards the young end of their age distribution. The goal of our study is to eliminate this bias. Measuring the true age distribution of nuclear star clusters will allow us to answer the question whether repeated nuclear starbursts are indeed common in late-type spirals, and, if so, what their duty cycle is. 


\section{Summary}

We have presented a new, high-quality spectrum of the nuclear star cluster of NGC 4449 which covers the region between 3700 and $9000 \AA$. Analysis of a variety of stellar absorption features leads to the conclusion that the nucleus of NGC 4449 has undergone a short-lived starburst about $6-10 \mathrm{Myr}$ ago. We do not find evidence for significant metal enrichment due to multiple previous periods of star formation in the nucleus of NGC 4449.

We thank the anonymous referee for useful comments that helped to improve the presentation of this paper. 


\section{REFERENCES}

Athanassoula, E. 1992a, MNRAS, 259, 328

Athanassoula, E. 1992b, MNRAS, 259, 345

Benjamin, R. A., Skillman, E. D., \& Smits, D. P. 1999, ApJ, 514, 307

Bica, E., Santos, J. F. C., Jr., \& Alloin, D. 1990, A\&A, 235, 103

Böker, T., et al. 1999a, ApJS, 124, 95

Böker, T., van der Marel, R. P., \& Vacca, W. D. 1999b, AJ, 118, 831

Bruzual, A. \& Charlot, S. 1993, ApJ, 405, 538

Calzetti, D. 1997, AJ, 113, 162

Cardelli, J. A., Clayton, G. C., \& Mathis, J. S. 1989, ApJ, 345, 245

Carollo, C. M., Stiavelli, M., \& Mack, J. 1998, AJ, 116, 68

Courteau, S., de Jong, R., \& Broeils, A. 1996, ApJL, 457, L73

de Vaucouleurs, G. 1948, Ann. d'Astrophysique, 11, 247

de Vaucouleurs, G., de Vaucouleurs, A., Corwin, H., Buta, R. J., Paturel, G., \& Fouque, P. 1991, Third Reference Catalogue of Bright Galaxies, New York:Springer-Verlag

Díaz, A. I., Terlevich, E., \& Terlevich, R. 1989, MNRAS, 239, 325

Frei, Z., Guhathakurta, P., Gunn, J. E., \& Tyson, J. A. 1996, AJ, 111, 174

Friedli, D. \& Benz, W. 1993, A\&A, 268, 65

García-Vargas, M. L., Mollá, M., \& Bressan, A. 1998, A\&AS, 130, 513

Gelatt, A. E., Hunter, D. A., \& Gallagher, J. S. 2001, PASP, in press (February issue)

González Delgado, R. M., Leitherer, C., \& Heckman, T. M. 1999, ApJS, 125, 489

Hill, R. S. et al. 1998, ApJ, 507, 179

Ho, L. C., Filippenko, A. V., \& Sargent, W. L. W. 1997, ApJS, 112, 315

Hubble, E. 1936, The Realm of the Nebula, Yale University Press, New Haven

Hummer, D. G., \& Storey, P. J. 1987, MNRAS, 224, 801

Hunter, D. A., van Woerden, H., \& Gallagher, J. S. 1999, AJ, 118, 2184

Kobulnicky, H. A., Kennicutt, R. C. Jr., \& Pizagno, J. L. 1999, ApJ, 514, 544 
Kormendy. J. \& Bender, R. 1996, ApJ, 464, 119

Kraan-Korteweg, R. C. \& Tamman, G. A. 1979, Astron. Nachr. 300, 181

Krabbe, A. et al. 1995, ApJ, 447, L95

Kriss, G. 1994, in ASP Conf. Ser. 61, Astronomical Data Analaysis Software and Systems III, ed. D. R. Crabtree, R. J. Hanisch, \& J. Barnes (San Francisco: ASP), 437

Lauer, T. R., Faber, S. M., Ajhar, E. A., Grillmair, C. J., \& Scowen, P. A. 1998, AJ, 116, 2263

Langer, N. \& Maeder, A. 1995, A\&A 295, 685

Leitherer, C., et al. 1999, ApJS, 123, 3

Lisenfeld, U. \& Ferrara, A. 1998, ApJ, 496, 145

Massey, P., Strobel, K., Barnes, J. V., \& Anderson, E. 1988, ApJ, 328, 315

Matthews, L. D. et al. 1999, AJ, 118, 208

Mayya, Y. D. 1994, AJ, 108, 1276

Merritt, D. \& Sellwood, J. A. 1994, ApJ, 425, 551

Norman, C. A., Sellwood, J. A., Hasan, H. 1996, ApJ, 462, 114

Oey, M. S., \& Shields, J. C. 2000, ApJ, 539, 687

Origlia, L., Goldader, J. D., Leitherer, C., Schaerer, D., \& Oliva, E. 1999, ApJ, 514, 96

Osterbrock, D. E. 1989, Astrophysics of Gaseous Nebulae and Active Galactic Nuclei, University Science Books:Mint Valley, CA

Pagel, B. E. J., Edmunds, M. G., Blackwell, D. E., Chun, M. S., \& Smith, G. 1979, MNRAS, 189, 95

Phillips, A.C., Illingworth, G. D., MacKenty, J. W., \& Franx, M. 1996, AJ, 111, 1566

Raha, A., Sellwood, J.A., James, R., \& Kahn, F.D. 1991, Nat, 352, 411

Rix, H.-W. \& White, S. D. M. 1992, MNRAS, 254, 389

Sabbadin, F., Ortolani, S., \& Bianchini, A. 1984, A\&A, 131, 1

Salpeter, E. E. 1955, ApJ, 121, 161

Sandage, A. 1961, The Hubble Atlas of Galaxies, Carnegie Institution, Washington

Sandage, A. \& Tamman, G. A. 1981, A Revised Shapley-Ames Catalog of Bright Galaxies, Carnegie Institution, Washington 
Schlegel, D. J., Finkbeiner, D. P., \& Davis, M. 1998, ApJ, 500, 525

Shields, J. C. \& Filippenko, A. V. 1992, ASP Conf. series, 31, 267

van den Bergh, S. 1995, AJ, 110, 613

Willmarth, D. \& Barnes, J. 1994, A User's Guide to Reducing Echelle Spectra with IRAF, National Optical Astronomy Observatories, Arizona

This preprint was prepared with the AAS $\mathrm{LAT}_{\mathrm{E}} \mathrm{X}$ macros v5.0. 
Fig. 1.- Ground-based R-band image of NGC 4449 from Frei et al. (1996). The field is $7^{\prime} \times 7^{\prime}$, with North up and East to the left. The greyscale has been optimized to the dynamic range of the galaxy. The nuclear cluster is clearly visible.

Fig. 2. - Top: spectrum of the nuclear star cluster of NGC 4449 , taken over a $2^{\prime \prime} \times 2^{\prime \prime}$ aperture. The underlying disk/bulge emission has been subtracted. Bottom: the same spectrum on an expanded y-scale. The residuals near $7580 \AA, 6280 \AA$, and $6860 \AA$ are due to imperfect correction of telluric oxygen absorption ("A-band"). A few sky lines have not been perfectly corrected, and appear as negative "spikes".

Fig. 3.- Normalized and rectified spectrum of the Balmer series (top) and the CaT absorption features (bottom). The horizontal bars denote the line and continuum windows that were used for measurement of the EW (Table 1).

Fig. 4. - Spectrum of $\mathrm{H} \delta$ before (top) and after (bottom) removal of the line emission. The Gauss and Voigt profile fits that were used to subtract the emission line and to judge the completeness of the line removal are overplotted in the respective panels (dashed lines).

Fig. 5.- Comparison of the Balmer EW with evolutionary synthesis models of González Delgado et al. (1999) for an instantaneous burst with a Salpeter (1955) IMF between $\mathrm{M}_{\text {low }}=1 \mathrm{M}_{\odot}$ and $\mathrm{M}_{\mathrm{up}}=80 \mathrm{M}_{\odot}$. The horizontal grey bar indicates the measured EW for the nuclear cluster in NGC 4449, its width corresponds to the measurement uncertainty. The crossing point of bar and model indicates an age of $\leq 10 \mathrm{Myr}$.

Fig. 6.- Comparison of the CaT EW with evolutionary synthesis models of García-Vargas et al. (1998) for an instantaneous burst with a Salpeter (1955) IMF between $\mathrm{M}_{\text {low }}=0.8 \mathrm{M}_{\odot}$ and $\mathrm{M}_{\text {up }}=100 \mathrm{M}_{\odot}$. The horizontal grey bar indicates the measured EW for the nuclear cluster in NGC 4449, its width corresponds to the measurement uncertainty. The crossing point of bar and model indicates an age of $\approx 10 \mathrm{Myr}$. The low-metallicity models are highly uncertain, the fact that they do not reproduce the observed EW should not be considered as evidence for a high cluster metallicity (see discussion in text).

Fig. 7.- Results of the spectral modeling. The modeled spectrum for a $10 \mathrm{Myr}$ stellar population with a Salpeter (1955) IMF, based on the Bruzual \& Charlot (1993) evolutionary tracks (thick line) is plotted over the observed spectrum of the nuclear cluster in NGC 4449. The spectrum has a resolution of $\approx 1 \AA$, about 5 times higher than the model. 
Table 1. Absorption features

\begin{tabular}{|c|c|c|c|}
\hline Species & Line window & $\mathrm{EW}[\AA]$ & Cont. window \\
\hline H10 $\lambda 3799$ & $3783-3813$ & 2.81 & $\mathrm{a}$ \\
\hline H9 $\lambda 3835$ & $3823-3853$ & 3.72 & $\mathrm{a}$ \\
\hline H8 $\lambda 3889$ & 3874-3904 & 3.96 & $\mathrm{a}$ \\
\hline $\mathrm{H} \epsilon \lambda 3970$ & 3955-3985 & 2.01 & $\mathrm{a}$ \\
\hline $\mathrm{H} \delta \lambda 4102$ & $4070-4130$ & 5.03 & $\mathrm{~b}$ \\
\hline $\mathrm{H} \gamma \lambda 4340$ & $4311-4371$ & 3.42 & c \\
\hline $\mathrm{H} \beta \quad \lambda 4862$ & $4830-4890$ & 3.88 & d \\
\hline Ca II $\lambda 8498$ & $8483-8513$ & 4.05 & e \\
\hline Ca II $\lambda 8542$ & $8527-8557$ & 6.78 & e \\
\hline CaII $\lambda 8662$ & $8647-8677$ & 4.45 & e \\
\hline
\end{tabular}

Note. - Continuum windows are defined as follows:

(a) $3740: 3743,3760: 3762,3782: 3785,3811: 3812,3909: 3915$

(b) 4019:4020,4037:4038,4060:4061,4138:4140,4148:4150

(c) 4301:4305,4310:4312,4316:4318,4377:4381,4392:4394,4397:4398

(d) $4820: 4830,4890: 4900$

(e) $8447-8462,8842-8857$

Windows (a)-(d) are taken from González Delgado et al.

(1999), (e) is from Díaz et al. (1989) 
Table 2. Emission Line Fluxes

\begin{tabular}{|c|c|c|c|}
\hline Species & $\mathrm{F}\left[10^{-15} \mathrm{ergs} / \mathrm{s} / \mathrm{cm}^{2}\right]$ & $\mathrm{F} / \mathrm{F}(\mathrm{H} \beta)$ & $\mathrm{F}_{\mathrm{c}} / \mathrm{F}_{\mathrm{c}}(\mathrm{H} \beta)$ \\
\hline$[\mathrm{O}$ II $] \lambda 3726$ & $42.3 \pm 0.6$ & $1.56 \pm 0.03$ & $2.27 \pm 0.05$ \\
\hline$[\mathrm{O}$ II] $\lambda 3729$ & $58.4 \pm 0.6$ & $2.15 \pm 0.04$ & $3.13 \pm 0.06$ \\
\hline H10 $\lambda 3798$ & $\leq 0.2$ & $\leq 0.01$ & - \\
\hline H9 $\lambda 3836$ & $0.9 \pm 0.2$ & $0.03 \pm 0.01$ & $0.04 \pm 0.02$ \\
\hline$[\mathrm{Ne}$ III $] \lambda 3869$ & $4.2 \pm 0.5$ & $0.15 \pm 0.02$ & $0.22 \pm 0.03$ \\
\hline H8 $\lambda 3889$ & $2.5 \pm 0.4$ & $0.09 \pm 0.01$ & $0.13 \pm 0.02$ \\
\hline $\mathrm{H} \epsilon \lambda 3970$ & $2.6 \pm 0.3$ & $0.1 \pm 0.01$ & $0.13 \pm 0.02$ \\
\hline $\mathrm{H} \delta \lambda 4102$ & $5.0 \pm 0.4$ & $0.18 \pm 0.02$ & $0.24 \pm 0.02$ \\
\hline $\mathrm{H} \gamma \lambda 4340$ & $11.0 \pm 0.3$ & $0.41 \pm 0.01$ & $0.48 \pm 0.01$ \\
\hline [O III] $\lambda 4363$ & $1.2 \pm 0.4$ & $0.04 \pm 0.01$ & $0.05 \pm 0.02$ \\
\hline $\mathrm{H} \beta \quad \lambda 4862$ & $27.1 \pm 0.4$ & $1.00 \pm 0.02$ & $1.00 \pm 0.02$ \\
\hline [O III] $\lambda 4959$ & $20.0 \pm 0.4$ & $0.74 \pm 0.02$ & $0.72 \pm 0.02$ \\
\hline$[\mathrm{O}$ III] $\lambda 5007$ & $58.9 \pm 0.4$ & $2.17 \pm 0.04$ & $2.04 \pm 0.03$ \\
\hline$[\mathrm{He} \mathrm{I}] \lambda 5876$ & $3.3 \pm 0.2$ & $0.12 \pm 0.01$ & $0.10 \pm 0.01$ \\
\hline$[\mathrm{O}$ I] $\lambda 6300$ & $2.9 \pm 0.4$ & $0.11 \pm 0.01$ & $0.08 \pm 0.01$ \\
\hline$[\mathrm{N}$ II $] \lambda 6548$ & $4.2 \pm 0.4$ & $0.15 \pm 0.01$ & $0.11 \pm 0.01$ \\
\hline $\mathrm{H} \alpha \lambda 6563$ & $109.5 \pm 0.3$ & $4.04 \pm 0.06$ & $2.82 \pm 0.04$ \\
\hline$[\mathrm{N}$ II $] \lambda 6583$ & $13.5 \pm 0.3$ & $0.5 \pm 0.01$ & $0.35 \pm 0.01$ \\
\hline$[\mathrm{S} \mathrm{II}] \lambda 6716$ & $13.0 \pm 0.3$ & $0.48 \pm 0.01$ & $0.32 \pm 0.01$ \\
\hline$[\mathrm{S}$ II $] \lambda 6731$ & $10.1 \pm 0.3$ & $0.37 \pm 0.01$ & $0.25 \pm 0.01$ \\
\hline$[$ Ar III $] \lambda 7136$ & $3.1 \pm 0.3$ & $0.11 \pm 0.01$ & $0.7 \pm 0.01$ \\
\hline
\end{tabular}

Note. - Column 2: Line flux as measured from Figure 1. Column 3: measured line ratio relative to $\mathrm{H} \beta$. Column 4: line ratio relative to $\mathrm{H} \beta$ after correction for an extinction of $\mathrm{A}_{\mathrm{V}}=1.1 \mathrm{mag}$. Uncertainties listed are $1 \sigma$. 


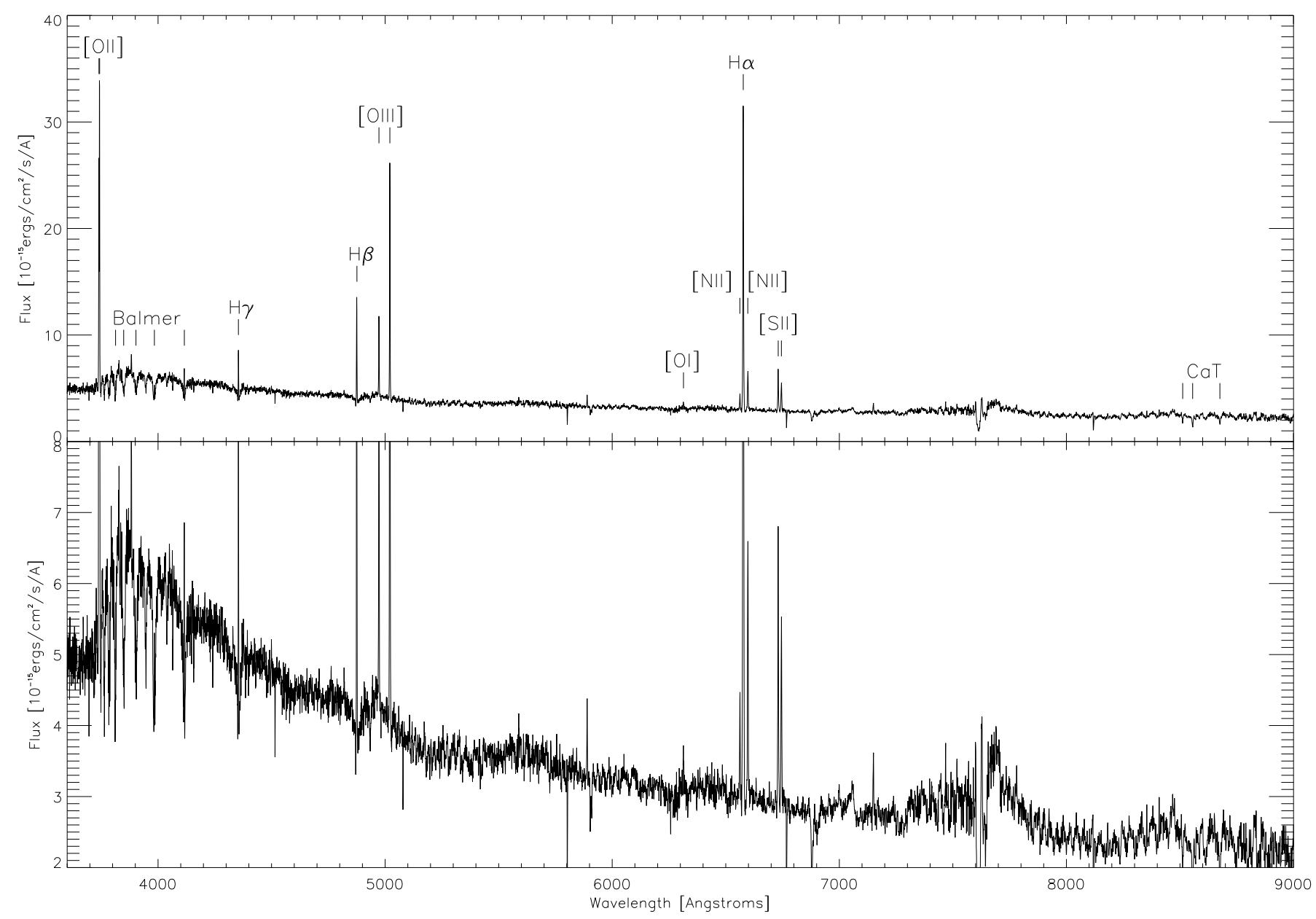



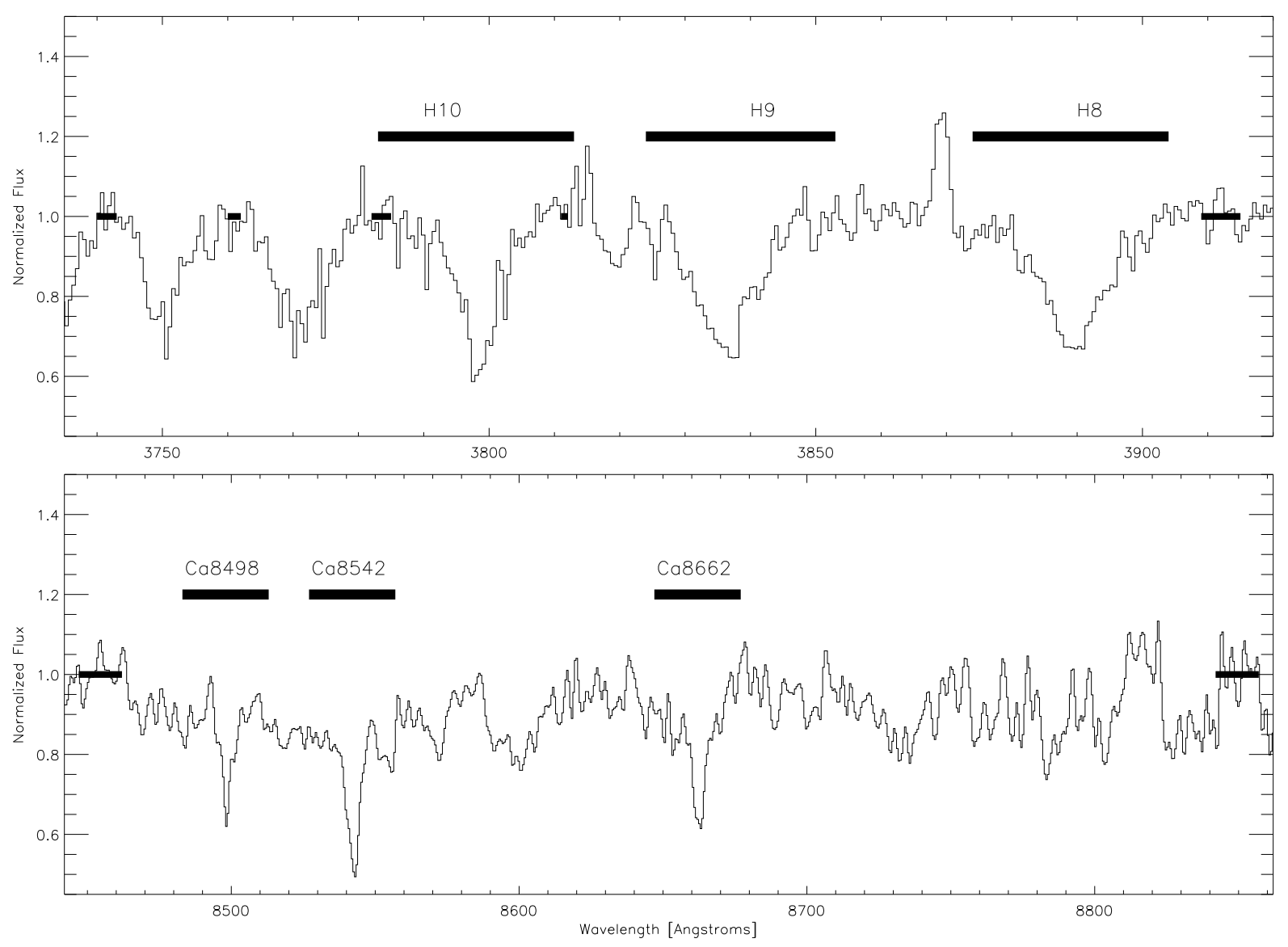


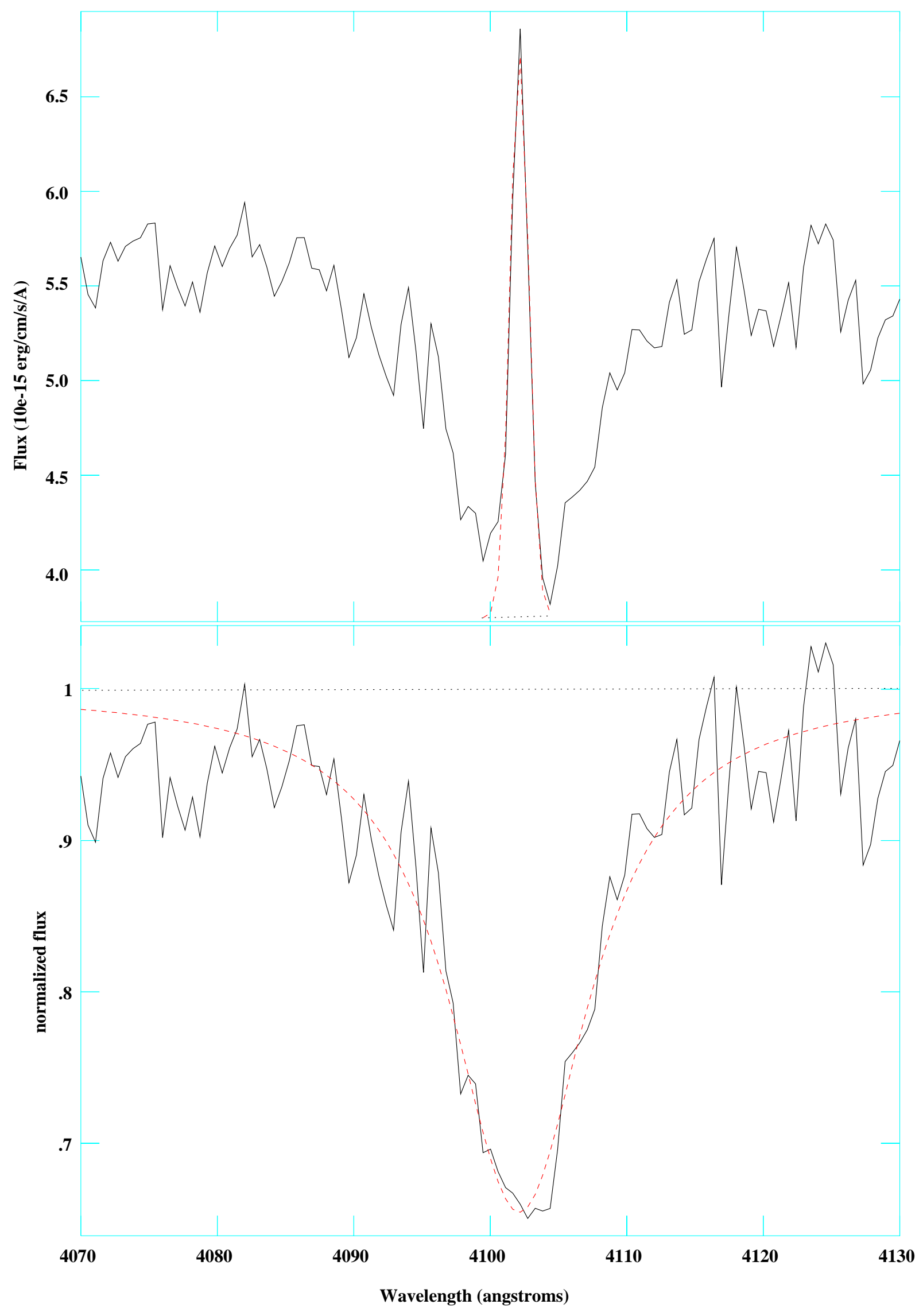




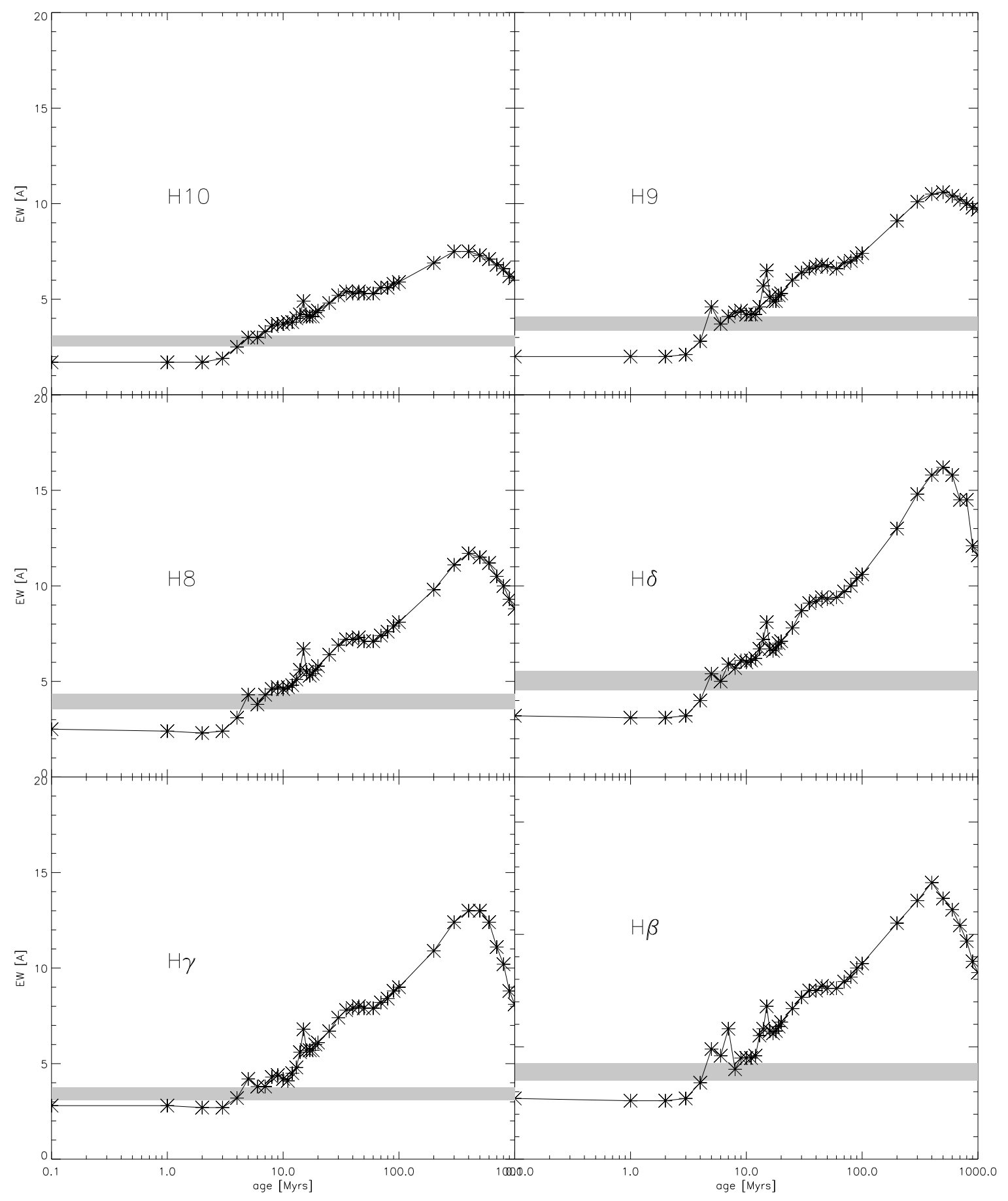




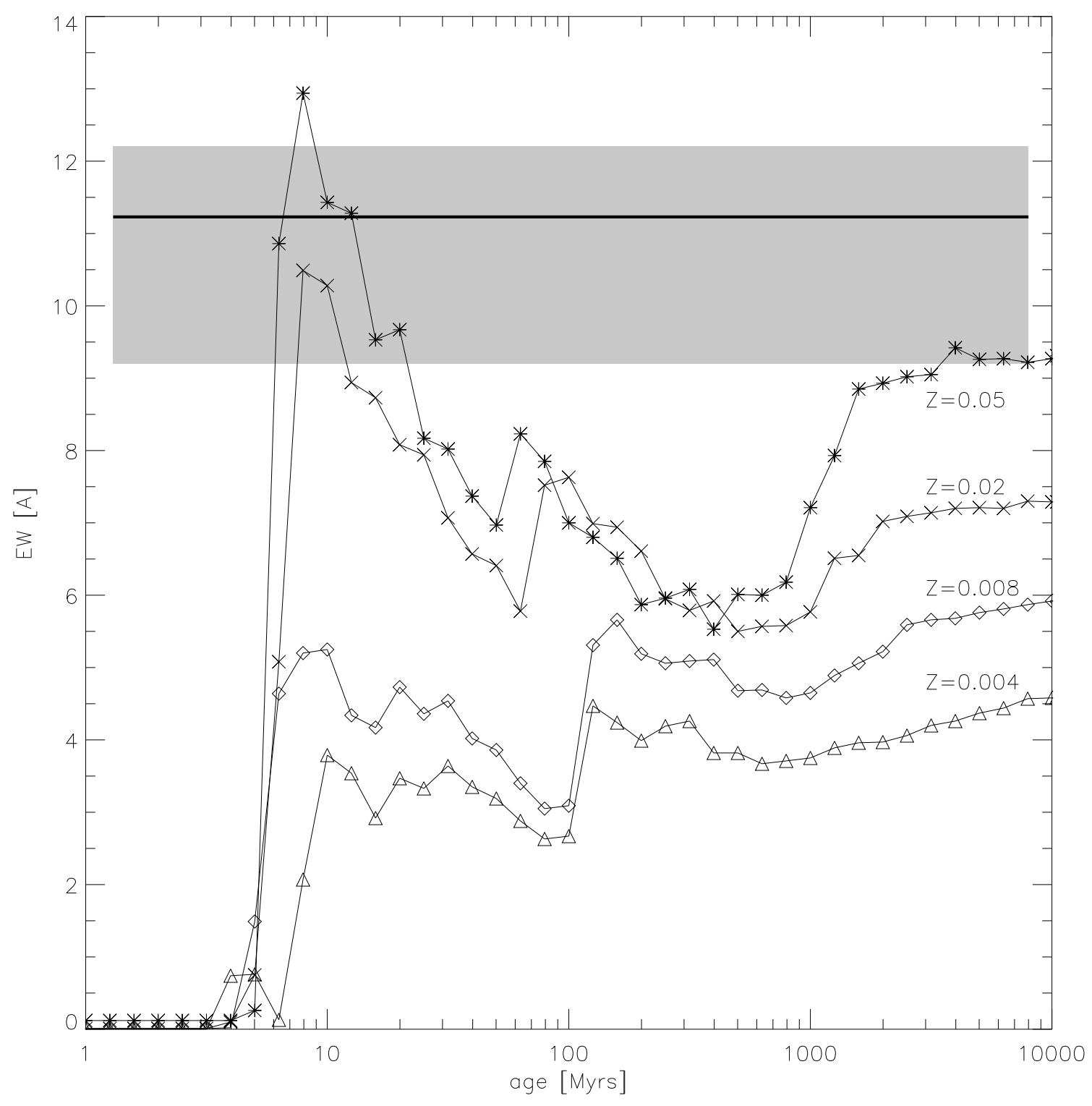




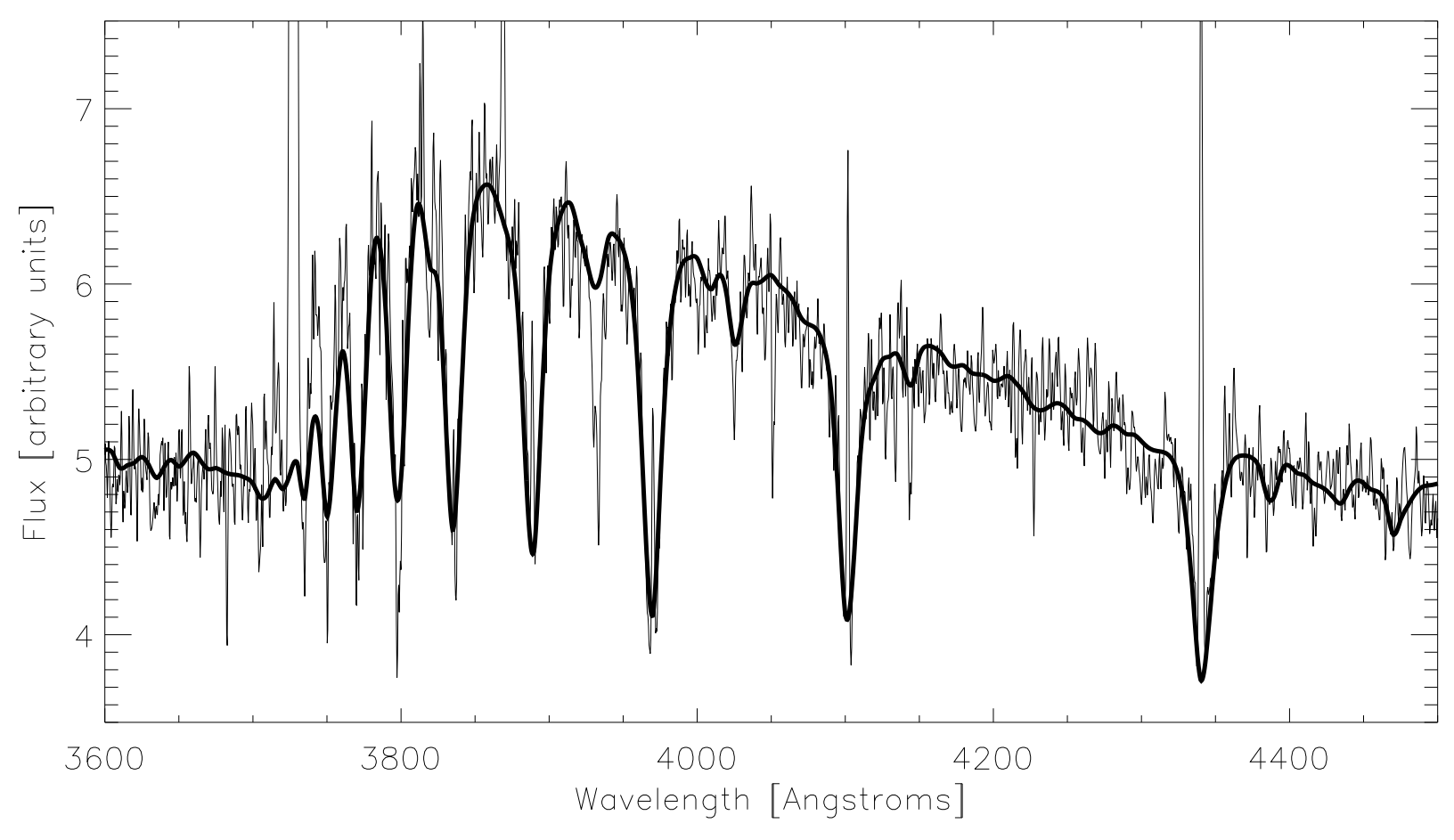


This figure "boeker.fig1.gif" is available in "gif" format from: http://arxiv.org/ps/astro-ph/0010542v2 